\title{
SRSF6 regulates alternative splicing of genes involved in DNA damage response and DNA repair in HeLa cells
}

\author{
XIAO YANG ${ }^{1}$, PENG ZHAN ${ }^{1}$, SHUQIANG FENG ${ }^{1}$, HE JI $^{2}$, WENJIE TIAN ${ }^{1}$, \\ MENGDI WANG ${ }^{3}, \mathrm{CHAO} \mathrm{CHENG}^{3}$ and $\mathrm{BIN} \mathrm{SONG}^{2}$ \\ Departments of ${ }^{1}$ Urology and ${ }^{2}$ Neurosurgery, The Second Hospital of Jilin University, \\ Changchun, Jilin 130041; ${ }^{3}$ ABLife BioBigData Institute, Wuhan, Hubei 430075, P.R. China
}

Received October 18, 2019; Accepted June 18, 2020

DOI: $10.3892 /$ or.2020.7750

\begin{abstract}
Alternative splicing (AS) occurs in nearly all human genes and abnormal AS has a close association with cancer. Serine and arginine-rich splicing factor 6 (SRSF6), a canonical member of the serine/arginine-rich protein family, has been characterized as an important regulator of AS. However, the role of SRSF6 in regulating AS in cancers has remained to be fully elucidated. In the present study, the median expression of SRSF6 in tumors was determined to be higher compared with that in matched normal tissues in 13 out of 16 cancer types from The Cancer Genome Atlas. To investigate the biological effects of SRSF6 overexpression, an SRSF6-overexpression model of HeLa cells was constructed and it was revealed that SRSF6 overexpression resulted in significantly higher apoptosis and lower proliferation compared to control cells. Transcriptome analysis indicated that overexpression of SRSF6 in cancer cells induced large-scale changes in transcriptional expression levels and AS. Two groups of cervical cancer tumor samples in which SRSF6 was differentially expressed were then selected to analyze potential SRSF6-regulated AS. It was determined that the pattern of SRSF6-regulated AS in clinical samples was similar to that in cancer cells and AS genes were enriched in DNA damage response (DDR) pathways, including DNA repair and double-strand break repair via homologous recombination. Furthermore, AS events regulated by SRSF6 were validated using reverse transcription-quantitative PCR. The present results highlighted that SRSF6 is able to trigger the activation of DDR pathways via regulation of $\mathrm{AS}$ to influence cancer progression. These results markedly expand the current understanding of the mechanisms underlying SRSF6-mediated gene regulation and suggest the potential use of SRSF6 as a therapeutic target in cancer.
\end{abstract}

Correspondence to: Dr Bin Song, Department of Neurosurgery, The Second Hospital of Jilin University, 2nd Building, 218 Pingzhi Street, Changchun, Jilin 130041, P.R. China

E-mail: 14683657@qq.com

Key words: SRSF6/SRP55, alternative splicing, cancer, DNA damage response, DNA repair

\section{Introduction}

Cancer is a major public health problem worldwide and is expected to be the leading cause of death and the single most important barrier to increasing life expectancy in the 21 st century (1). It is now understood that advanced age and exposure to ionizing radiation may be the most important risk factors promoting carcinogenesis $(2,3)$. Substantial research progress has been made in recent years to understand the basic mechanisms underlying cancer initiation and progression and to identify potential targets for therapeutic intervention. Thanks to the advance of next-generation sequencing technology, extensive transcriptome and genome analyses of cancer have identified multiple recurrently altered genes and pathways by robustly assessing RNA sequencing (RNA-seq) and whole exome sequencing data of tumor and matched normal tissue samples $(4,5)$. These pathways include the PI3K/AKT signaling pathway, the Wnt signaling pathway and the DNA damage response (DDR) pathway (6-8). Although errors in the DDR contribute to genome instability, which may lead to tumor progression, they also provide therapeutic opportunities $(9,10)$. In particular, the involvement of post-transcriptional regulation within the DDR pathway is now becoming evident (11).

Post-transcriptional regulation is important for modulation of gene expression and involves numerous different aspects of biological processes, including RNA metabolism, pre-mRNA splicing and translation (12). It is widely recognized that alternative splicing (AS) occurs in nearly all human genes and has a close connection to cancer-associated pathways $(13,14)$. Compared to normal tissues, tumors present with abnormal splicing patterns that have a pathogenic role in cancer progression (15-18). For instance, AS of Cyclin D1 generates two isoforms, Cyclin D1a and D1b. Cyclin D1b is upregulated in several types of cancer and is likely responsible for anchorage-independent growth of tumor cells, which highlights the role of AS in promoting sustained proliferation signals in cancer (19-21).

A number of factors involved in AS regulation have been well characterized, including serine/arginine-rich (SR) RNA-binding proteins. SR proteins, also called SR splicing factors (SRSF), contain one or two RNA-recognition motifs functioning primarily in RNA binding and an SR domain 
enriched in arginine and serine involved in protein-protein interactions $(22,23)$. There are 12 canonical members of the SR protein family that share this classical domain structure (SRSF1-12). SR proteins have been extensively characterized for their activities in regulating constitutive and alternative pre-mRNA splicing (24). Abnormal expression of SR proteins has been reported in various cancer types, including leukemia $(25,26)$, as well as breast (27), colon (28), skin (29), colorectal (30) and lung (28) cancer, and SRSF1, SRSF3 and SRSF6 are always overexpressed in cancers (30-33).

SRSF6 is also known as SRP55 or SFRS6. In Drosophila, its B52 homologue is able to influence cell growth and the cell cycle, but not differentiation. Overexpression of B52 promotes cell growth and upregulates Myc transcription (34). SRSF6 also acts as a proto-oncogene, which is frequently overexpressed in human skin cancer, and its overexpression in transgenic mice produces hyperplasia of sensitized skin and promotes aberrant AS (29). A study from 2016 demonstrated that long intergenic non-protein coding RNA 1133 inhibits the endothelial-mesenchymal transition and metastasis by directly binding to SRSF6 as a target mimic, and thus, SRSF6 may serve as a prognostic biomarker and effective therapeutic target for colorectal cancer (35). Another study revealed that SRSF6 is frequently upregulated in colorectal cancer samples and is associated with poor prognosis, and confirmed its function in promoting proliferation and metastasis (30). This evidence supports the robust potential of SRSF6 protein to be useful biomarkers for diagnosis and prognosis or effective therapeutic targets in cancer. However, a comprehensive investigation of the transcriptional and post-transcriptional regulation of SRSF6 in cancer remains to be performed.

The present study extensively evaluated the expression levels of SRSF6 in 16 cancer types available in The Cancer Genome Atlas (TCGA) database, demonstrating an increase of SRSF6 in cancers compared to normal tissues in most types of cancer. To obtain further insight into how SRSF6 regulates gene transcription and its involvement in cancer progression, an SRSF6-overexpressing cell model was constructed. Overexpression of SRSF6 was revealed to promote apoptosis and inhibit cell proliferation. Using unbiased transcriptome analysis, the present results indicated that overexpression of SRSF6 in cancer cells induced AS of pre-mRNAs of hundreds of genes and also changed transcript profiles. As an RNA binding protein and splicing factor, SFRS6 has numerous predicted regulated targets, some of which are enriched in 'DNA repair' and the 'double-strand break repair via the homologous recombination pathway', as indicated by Gene Ontology (GO) term analysis. Subsequently, 16 cervical tumor samples, including 8 samples with high SRSF6 expression and 8 samples with low SRSF6 expression, were selected to further study the potential impact of SRSF6 on AS regulation of the cancer transcriptome. The SRSF6-regulated AS events (ASEs) that had been detected in cancer cells were also validated in those cervical cancer samples. Collectively, these results demonstrated that SRSF6 is able to regulate the transcriptome of genes involved in cancer progression by mediating gene expression and AS. The present study enhances the current understanding of the biological functions and regulatory roles of SRSF6.

\section{Materials and methods}

Retrieval and analysis of TCGA data. Analysis of TCGA data was performed with GEPIA (36). RNA-seq expression data of 308 cervical tumor samples were downloaded from TCGA to determine the expression levels of SRSF6. A total of 8 samples with high SRSF6 expression and 8 samples with low SRSF6 expression were then selected and their RNA-seq data were downloaded to analyze the regulation of alternative splicing in cervical cancer.

SRSF6 overexpression. Primer pairs used for Hot Fusion were designed by CE Design v1.04 (Vazyme Biotech Co., Ltd) with gene-specific sequences and they included a portion of vector pIRES-hrGFP-1a sequences, with a 17-30 bp overlap between primer pairs. The primer sequences were as follows: Forward, 5'-AGCCCGGGCGGATCCGAATTCATGCCG CGCGTCTACATAGG-3' and reverse, 5'-GTCATCCTTGTA GTCCTCGAGATCTCTGGAACTCGACCTGGACC-3'. The SRSF6-overexpressing plasmid was constructed and HeLa cells were transfected with SRSF6-overexpressing plasmid and empty plasmid as described in a previous study by our group (37).

$R T-q P C R$. The housekeeping gene GAPDH was utilized as a control gene to assess whether SRSF6 was overexpressed. Complementary (c)DNA synthesis was performed by RT using the Kit One-Step gDNA Removal and cDNA synthesis mix (cat. no. AT311-02; Transgen Biotech) at $65^{\circ} \mathrm{C}$ for $5 \mathrm{~min}, 25^{\circ} \mathrm{C}$ for $10 \mathrm{~min}$ and $42^{\circ} \mathrm{C}$ for $30 \mathrm{~min}$. qPCR was then performed using the Hieff ${ }^{\mathrm{TM}}$ qPCR SYBR ${ }^{\circledR}$ Green Master Mix (Low Rox Plus; YEASEN) in a Mycycler (Bio-Rad Laboratories) with the following thermocycling conditions: $95^{\circ} \mathrm{C}$ for $5 \mathrm{~min}$, followed by 40 cycles of $95^{\circ} \mathrm{C}$ for $10 \mathrm{sec}$ and $60^{\circ} \mathrm{C}$ for $30 \mathrm{sec}$. Primer sequences are listed in Table SI. The concentration of each transcript was then normalized to the level of GAPDH mRNA using the $2^{-\Delta \Delta \mathrm{Cq}}$ method (38). Comparisons were performed by a paired Student's t-test using GraphPad Prism software (v8.0; GraphPad Software, Inc.). RT-qPCR was also performed in the present study for certain selected RASEs, normalized to the reference gene GAPDH. The primers for detecting pre-mRNA splicing are listed in Table SI. To quantitatively analyze the two different splicing isoforms of a specific ASE using a qPCR approach, two pairs of primers were designed to specifically amplify each of these two isoforms after the initial synthesis of the first-strand cDNA using random primers (cat. no. AT311-02; Transgen). To achieve this specificity, a primer complementary to the splice junction of the constitutive exon and alternative exon was designed. The RNA samples used for RT-qPCR were the same as those used for RNA-seq. The RT step was the same as above. The PCR mixture was as described above. The PCR conditions consisted of denaturation at $95^{\circ} \mathrm{C}$ for $10 \mathrm{~min}$, followed by 40 cycles of denaturation at $95^{\circ} \mathrm{C}$ for $15 \mathrm{sec}$, and annealing and extension at $60^{\circ} \mathrm{C}$ for $1 \mathrm{~min}$ each. PCR amplifications were performed in triplicate for control and SRSF6 overexpression (SRSF6-OE) samples and quantified using $2^{-\Delta \Delta \mathrm{Cq}}$ method (38).

Western blot analysis. Proteins were extracted from samples by lysis with wash buffer (1X PBS, $0.1 \%$ SDS, $0.5 \%$ 
nonidet-P-40 and $0.5 \%$ sodium deoxycholate) and the protein concentration was determined using the bicinchoninic acid (BCA) method (BCA detection Kit; cat. no. P0012; Beyotime Institute of Biotechnology). Protein samples (20 $\mu \mathrm{g}$ per lane) were loaded onto 10 or $12 \%$ SDS-PAGE gels depending on the molecular weight which was determined using protein marker and then transferred onto $0.45-\mathrm{mm}$ polyvinylidene difluoride (PVDF) membranes (cat. no. ISQE00010; EMD Millipore). The PVDF membranes were then blocked with $5 \%$ skimmed milk (in a buffer containing $10 \mathrm{mM}$ Tris, $\mathrm{pH} 8.0,150 \mathrm{mM} \mathrm{NaCl}$ and $0.05 \%$ Tween-20) for $1 \mathrm{~h}$ at room temperature. Subsequently, they were incubated with primary antibody at $4^{\circ} \mathrm{C}$ overnight and then incubated with horseradish peroxidase-conjugated secondary antibody for $1 \mathrm{~h}$ at room temperature. The membranes were visualized through chemiluminescence reagent (cat. no. 32106; Thermo Fisher Scientific, Inc.) and the is ChemiScope imaging system from Clina. Protein bands were quantified using Image $\mathbf{J}$ software (v 1.8.0; National Institutes of Health). The following primary antibodies were used: anti-SRSF6 (1:1,000 dilution; polyclonal antibody; cat. no. A0511; AB Clonal) and anti-actin (1:1,000 dilution; polyclonal antibody; cat. no. AC001; ABClonal). The following secondary antibody was used: Horseradish peroxidase-conjugated goat anti-rabbit $\operatorname{IgG}$ (1:1,000 dilution; cat. no. AS014; ABClonal).

Cell proliferation and apoptosis assay. Cell proliferation was assessed using an MTT assay. In total, $5 \times 10^{3} \mathrm{HeLa}$ cells/well were cultured in 96-well plates. The cells were transfected with the SRSF6-overexpressing plasmid using Lipofectamine ${ }^{\circledR}$ 2000 according to the manufacturer's protocol. After incubation at $37^{\circ} \mathrm{C}$ for $48 \mathrm{~h}$, MTT solution $(0.025 \mathrm{ml}$ at $5 \mathrm{mg} / \mathrm{ml})$ was added to each well. The cells were incubated for another $4 \mathrm{~h}$ and the supernatant was removed from each well. The colored formazan crystals produced by MTT were dissolved in DMSO $(0.15 \mathrm{ml})$ and the optical density was measured using an ultraviolet analyzer (Bio-Rad Laboratories, Inc.) at $490 \mathrm{~nm}$.

For the flow cytometric analysis of cell apoptosis, the transfected cells were incubated at $37^{\circ} \mathrm{C}$ for $48 \mathrm{~h}$ and the live cells were then harvested and washed twice with ice-cold PBS. Viable cells were double-stained with 7-amino actinomycin D and FITC-conjugated Annexin V (Beijing 4A Biotech Co., Ltd.). The percentage of apoptotic cells was calculated as the sum of the right lower and upper quadrants. The number of stained cells was quantified using a flow cytometer (CytoFLEX; Beckman Coulter, Inc.). Cell cycle distribution was quantified using multi-cycle software (FlowJo 10.5.3; FlowJo, LLC).

RNA extraction and high-throughput sequencing. Total RNA was extracted using TRIzol reagent and was further purified with two phenol-chloroform treatments. To remove DNA, the purified RNA was then treated with RNase-free RQ1 DNase (Promega Corp.) and its quality and quantity were determined by measuring the absorbance at 260/280 nm (A260/A280) using a Smartspec Plus (Bio-Rad Laboratories, Inc.). The integrity of RNA was then verified by $1.5 \%$ agarose gel electrophoresis.

A total of $10 \mu \mathrm{g}$ total RNA from each sample was used to prepare a directional RNA-seq library. First, the polyadenylated
mRNAs were concentrated with oligo (dT)-conjugated magnetic beads (Invitrogen; Thermo Fisher Scientific, Inc.). The concentrated mRNAs were then iron-fragmented at $95^{\circ} \mathrm{C}$, end-repaired and ligated to a $5^{\prime}$ adaptor. RT was performed with RT primer harboring a $3^{\prime}$ adaptor sequence and randomized hexamer. The purified cDNAs were amplified and stored at $-80^{\circ} \mathrm{C}$ until they were used for sequencing. Following the manufacturer's instructions, the libraries were prepared for high-throughput sequencing. The Illumina HiSeq X Ten system (Illumina, Inc.) was used to collect data from 150-bp pair-end sequencing (BGI Inc.).

RNA-seq raw data clean and alignment. Raw sequencing reads containing more than $2-\mathrm{N}$ bases were first discarded. Subsequently, the raw reads were trimmed of adaptors and low-quality bases using a FASTX-Toolkit (v.0.0.13; http://hannonlab.cshl.edu/fastx_toolkit/). In addition, short reads of less than $16 \mathrm{nt}$ were dropped to retain clean reads, which were subsequently aligned to the GRch 38 genome by Tophat2 (39) with 4 mismatches. Uniquely mapped reads were ultimately used to calculate read number and paired-end fragments per kilobase of exon per million fragments mapped (FPKM) for each gene.

Differentially expressed gene (DEG) analysis. The expression levels of genes were evaluated using FPKM. The software edgeR (40), which is specifically used to analyze the differential expression of genes, was applied to evaluate the FPKM value and screen the RNA-seq data for DEGs. The results were analyzed based on the fold change $(\mathrm{FC} \geq 2$ or $\leq 0.5)$ and false discovery rate $(\mathrm{FDR}<0.05)$ to determine whether a gene was differentially expressed.

Alternative splicing analysis. The ABLas pipeline as described previously (41) was used to define and quantify the ASEs and regulated ASEs (RASEs) between the samples. In brief, detection of seven types of canonical ASEs in each sample was based on the splice junction reads. These ASEs were exon skipping (ES), exon included [cassette exon (CE)], alternative 5' splice site (A5SS), alternative 3' splice site (A3SS), mutual exclusive exon skipping (MXE), MXE combined with an alternative polyadenylation site and MXE combined with an alternative 5' promoter. Subsequently, the significant P-value was calculated using Fisher's exact test, with the model reads of samples and alternative reads as input data. The changed ratio of alternatively spliced reads and constitutively spliced reads between compared samples, which was defined as the RASE ratio, was calculated. A RASE ratio $>0.2$ and $\mathrm{P}<0.05$ were set as the threshold for RASE detection.

Functional enrichment analysis. Using the KOBAS 2.0 server (42), GO analyses and enriched Kyoto Encyclopedia of Genes and Genomes (KEGG) pathways were identified to predict the functions of genes and calculate the distribution frequency in each functional category. The enrichment of each pathway (corrected $\mathrm{P}<0.05$ ) was defined using hypergeometric tests and the Benjamini-Hochberg FDR controlling procedure. Reactome (http://reactome.org) pathway profiling was also used for functional enrichment analysis of the sets of selected genes. 


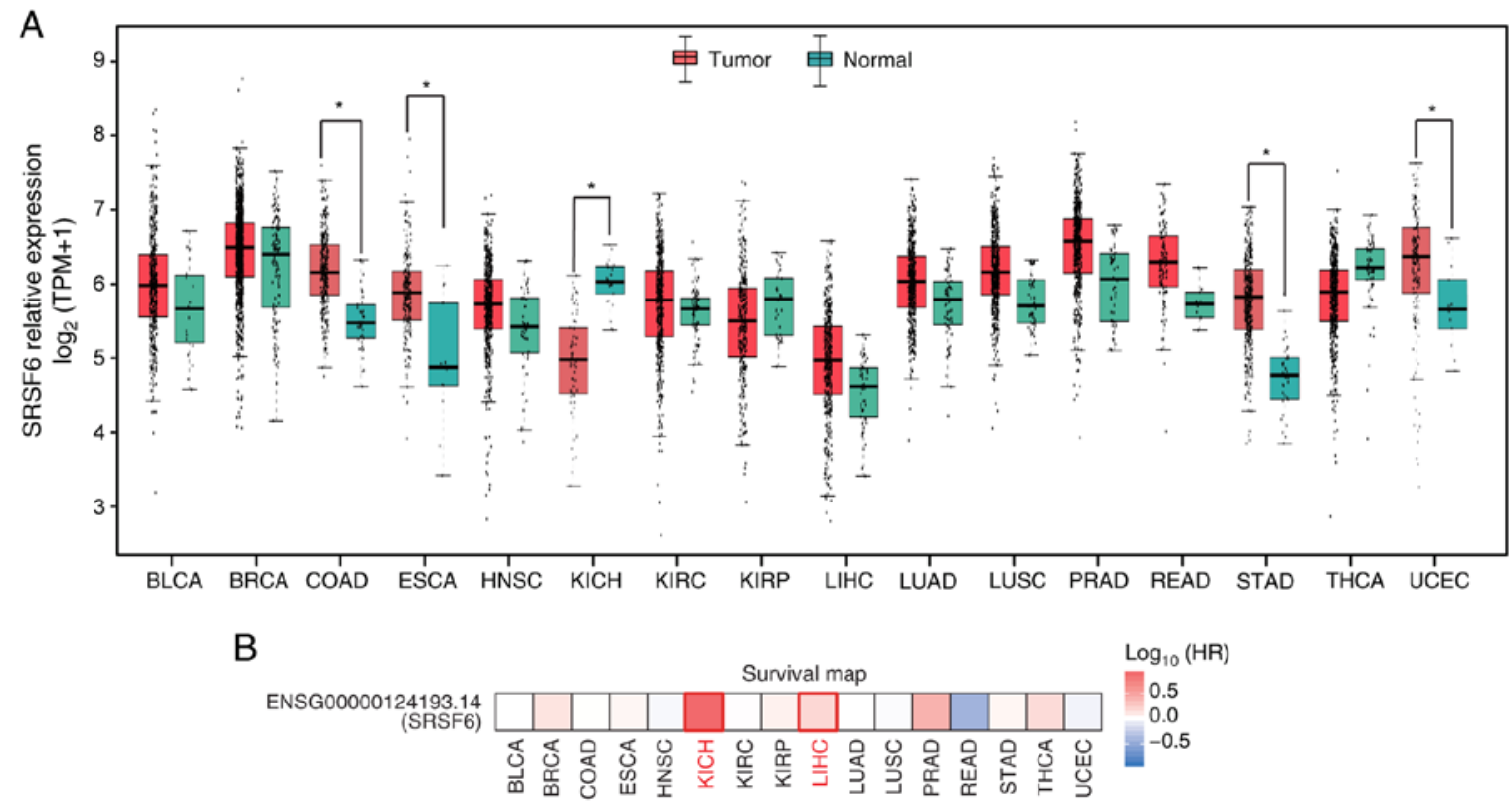

Figure 1. Differential expression of SRSF6 in tumor samples from The Cancer Genome Atlas. (A) Relative expression (TPM) of SRSF6 in tumor samples (red) compared with normal samples (green) from 16 cancer types. "P<0.05. (B) Association of SRSF6 expression with the survival rates in various types of cancer. SRSF6, serine and arginine-rich splicing factor 6; HR, hazard ratio; TPM, transcripts per million; BLCA, bladder urothelial carcinoma; BRCA, breast invasive carcinoma; COAD, colon adenocarcinoma; ESCA, esophageal carcinoma; HNSC, head and neck squamous cell carcinoma; KICH, kidney chromophobe; KIRC, kidney renal clear-cell carcinoma; KIRP, kidney renal papillary cell carcinoma; LIHC, liver hepatocellular carcinoma; LUAD, lung adenocarcinoma; LUSC, lung squamous-cell carcinoma; PRAD, prostate adenocarcinoma; READ, rectum adenocarcinoma; STAD, stomach adenocarcinoma; THCA, thyroid carcinoma; UCEC, uterine corpus endometrial carcinoma.
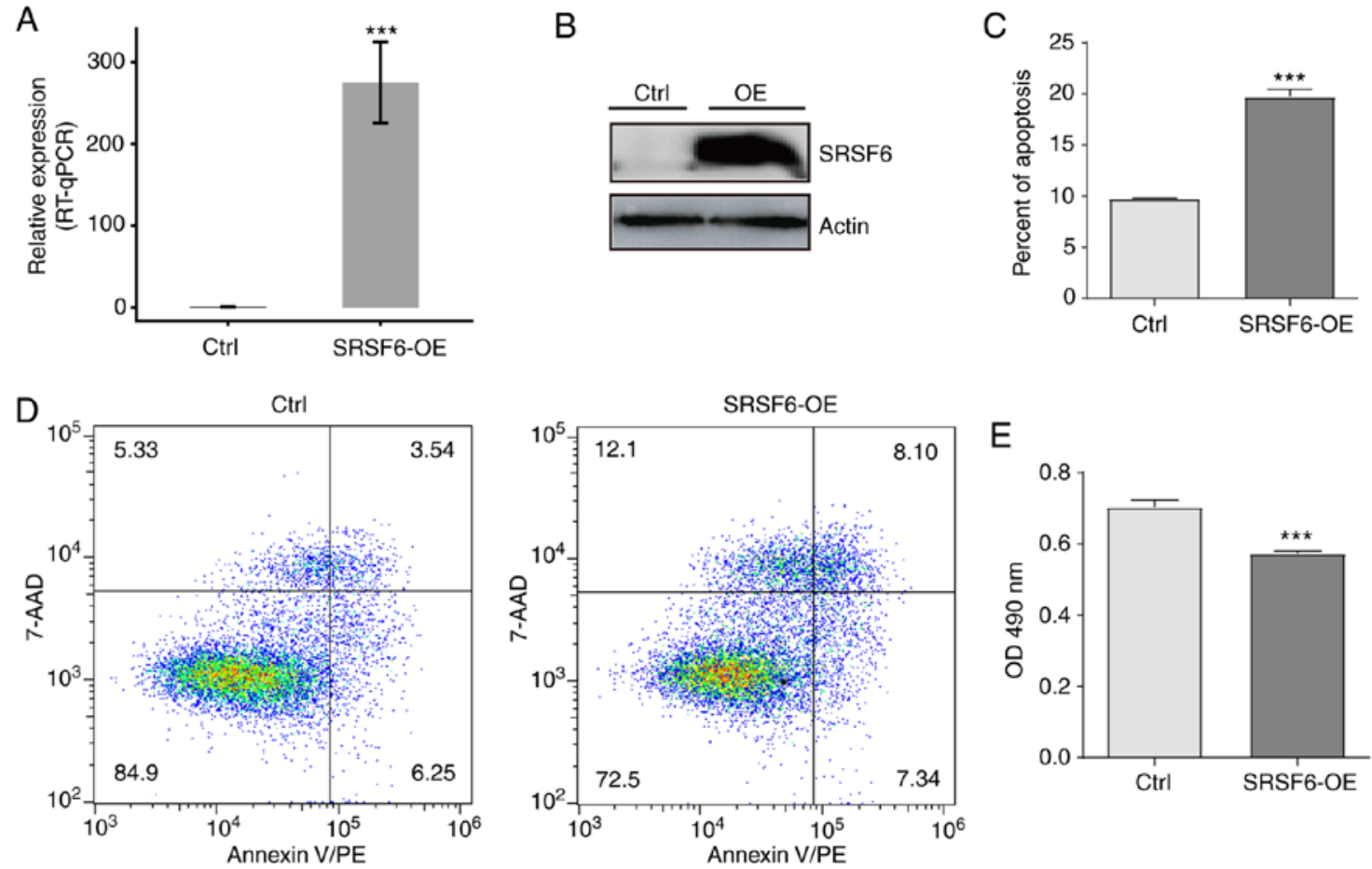

Figure 2. SRSF6 promotes HeLa cell apoptosis and inhibits cell proliferation. (A) Relative mRNA expression of SRSF6 was validated by RT-qPCR in HeLa cells after it was overexpressed. (B) The level of FLAG-tagged SRSF6 protein expression was determined by western blot analysis using $\beta$-actin as a housekeeping protein. (C and D) Apoptosis of SRSF6-overexpressing cells and controls was measured by flow cytometry following 7-AAD and annexin V staining. (C) Quantitative results and (D) representative flow cytometry dot plots. (E) Cell proliferation of SRSF6-overexpressing cells and controls was measured by MTT assay. ${ }^{* * *} \mathrm{P}<0.001$. SRSF6, serine and arginine-rich splicing factor 6; RT-qPCR, reverse transcription-quantitative PCR; OE, overexpression; Ctrl, control; PE, phycoerythrin; 7-AAD, 7-aminoactinomycin; OD, optical density.

Statistical analysis. One-way analysis of variance was used for comparison of expression levels of SRSF6 among
16 TCGA cancer types with GEPIA2, using disease state (Tumor or Normal) as the variable for calculating differential 
A

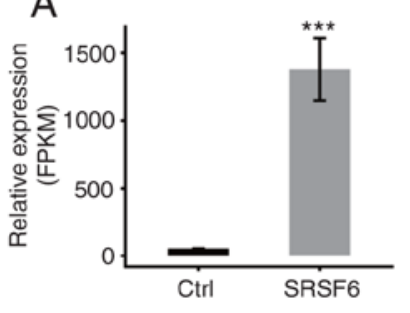

B

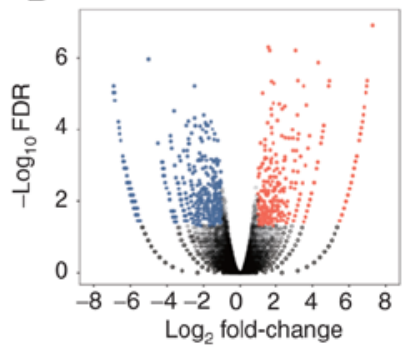

C

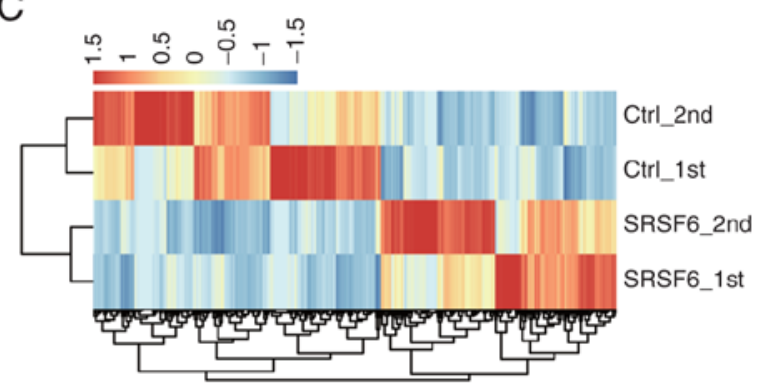

E
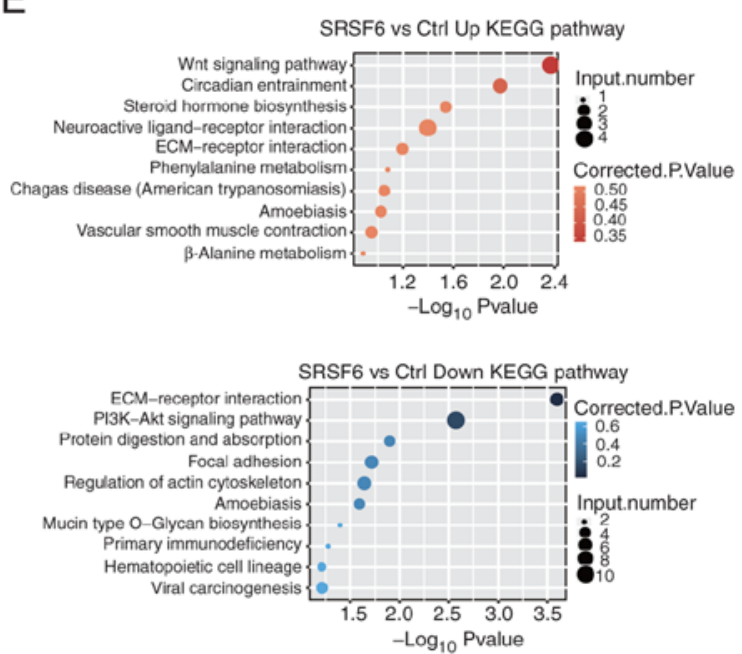

$-\log _{10}$ Pvalue
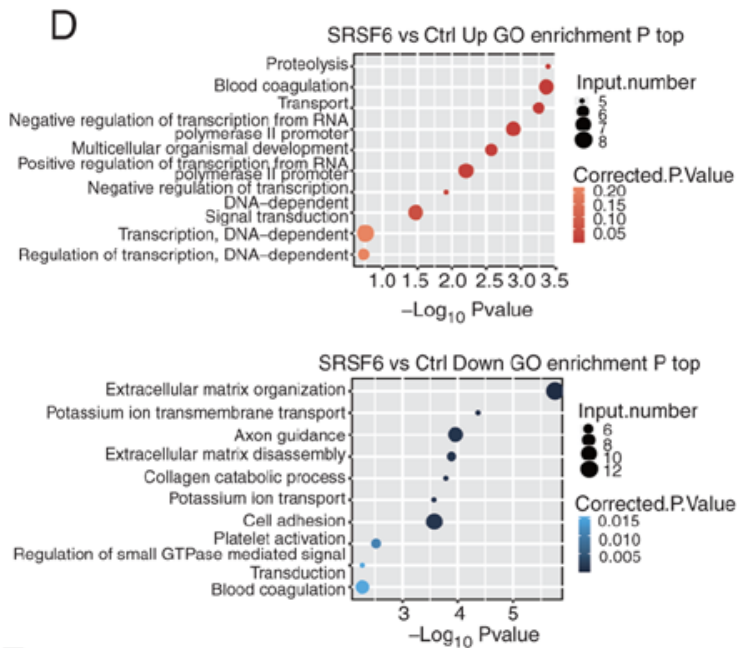

$\mathrm{F}$

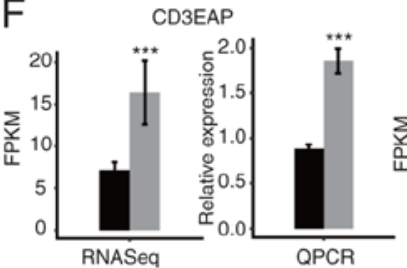

DLEU2L
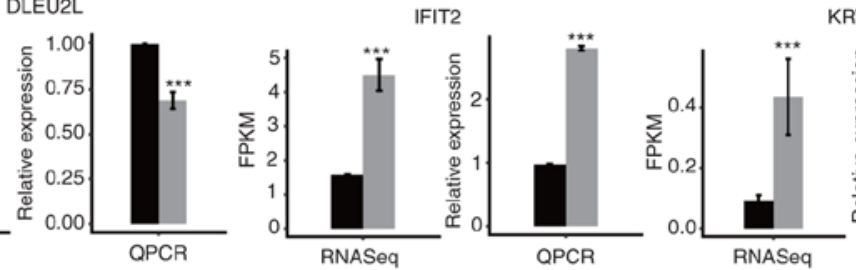

KRT13
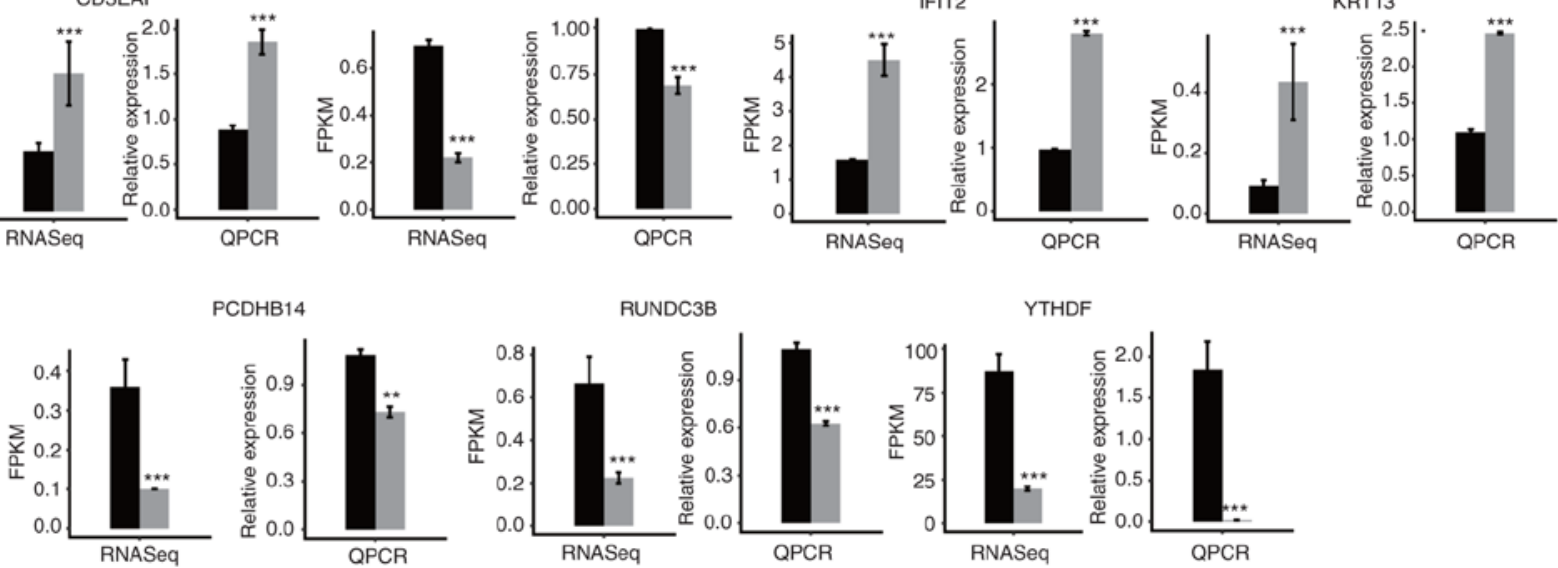

Figure 3. RNA-seq analysis of gene expression regulated by SRSF6 overexpression. (A) Following SRSF6 overexpression the mRNA expression level of SRSF6 was measured by RNA-seq and FPKM values were calculated. (B) Volcano plot of the genes regulated by SRSF6; upregulated genes (FC $\geq 2$; FDR $<0.05$ ) are labeled in red and downregulated genes $(\mathrm{FC} \leq-2 ; \mathrm{FDR}<0.05)$ are labeled in blue. (C) Heatmap of 837 DEGs between SRSF6 overexpression and control samples. Expression levels (FPKM) were log2-transformed and then median-centered for each gene. (D and E) The top 10 representative (D) GO biological process terms and (E) KEGG pathways of upregulated and downregulated genes following SRSF6 overexpression. (F) Reverse transcription-qPCR validation of DEGs regulated by SRSF6 in cancer cells; black bars are for the control group and grey bars for SRSF6 overexpression. ${ }^{* * *} \mathrm{P}<0.001$. Ctrl_1st and Ctrl_2nd, SRSF6_1st and SRSF6_2nd are two biological replicates. RNA-seq, RNA sequencing; SRSF6, serine and arginine-rich splicing factor 6; FPKM, fragments per kilobase of transcript per million mapped reads; qPCR, quantitative PCR; FC, fold change; FDR, false discovery rate; Ctrl, control; Up/Down, up-/downregulated genes; KEGG, Kyoto Encyclopedia of Genes and Genomes; ECM, extracellular matrix; GO, gene ontology; DEG, differentially expressed gene; POLR1G, RNA polymerase I subunit G; DLEU2L, deleted in lymphocytic leukemia 2 like; IFIT2, interferon induced protein with tetratricopeptide repeats 2; KRT13, keratin 13; PCDHB14, protocadherin beta 14; RUNDC3B, RUN domain containing 3B; YTHDF1, YTH N6-methyladenosine RNA binding protein 1.

expression. Student's t-test was used for all other comparisons between the SRSF6-OE and control groups. For each assay, the results are presented as the mean \pm standard error of the mean of three experiments. The data were analyzed using R software (v3.5.3, https://www.r-project.org/). $\mathrm{P}<0.01$ or FDR $<0.05$ was considered to indicate a statistically significant difference.

\section{Results}

Differential expression of SRSF6 in 16 cancer types from TCGA. Inspired by previous discoveries on the overexpression of SRSF6 in lung, breast, skin and colon cancer samples (31), SRSF6 expression data for 16 cancer types from TCGA which had at least 10 normal samples were analyzed using 
A
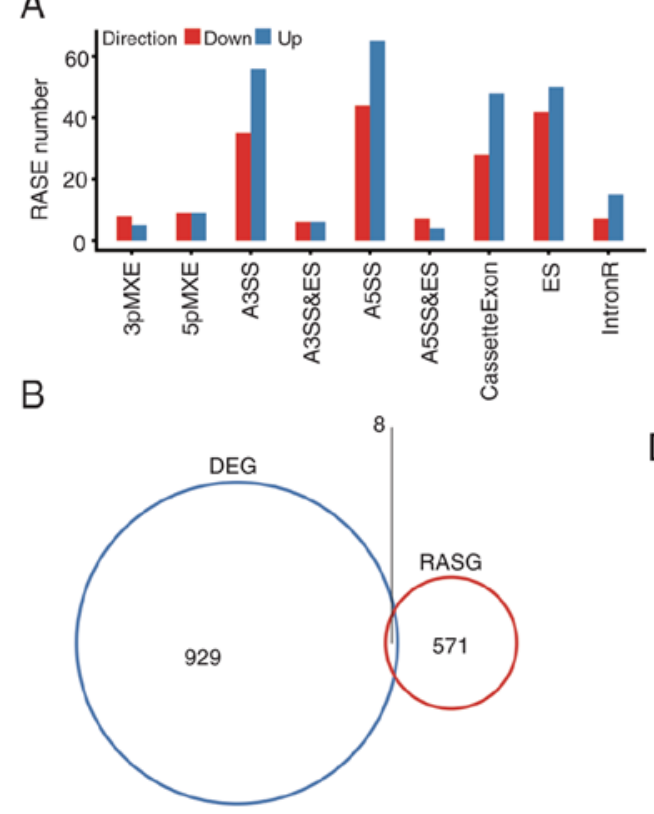

C

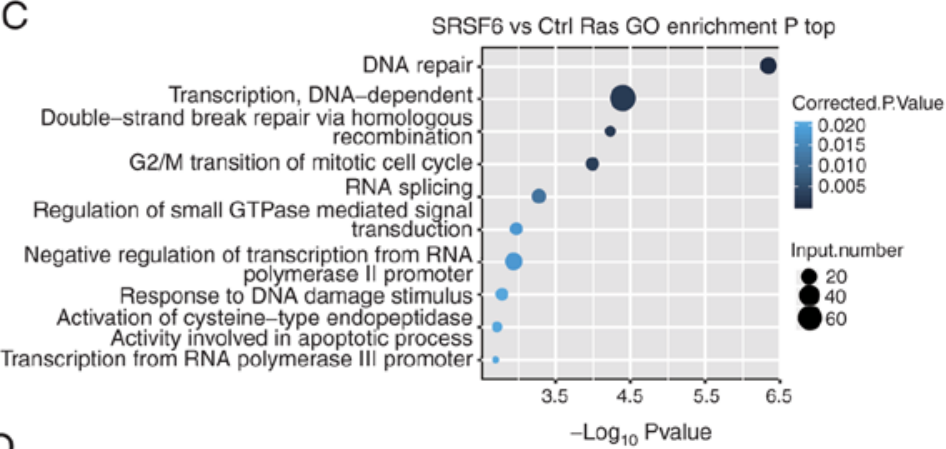

D

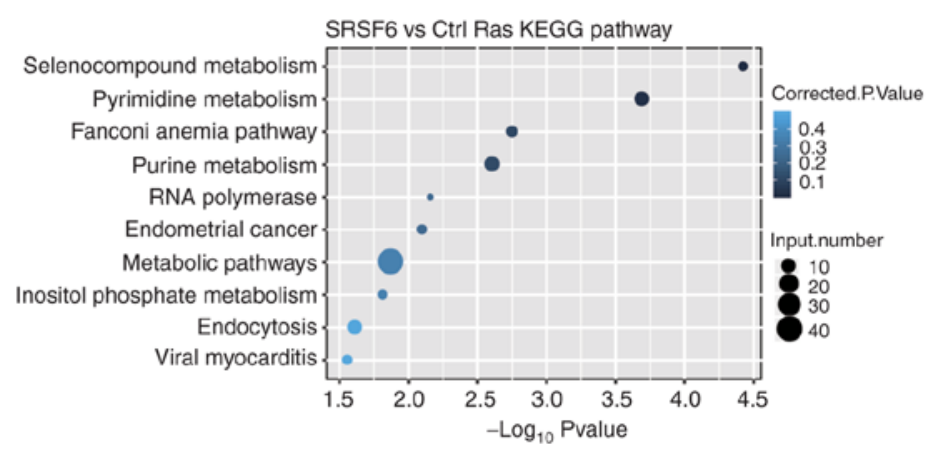

Figure 4. AS analysis of cancer cells. (A) Classification of differential AS types regulated by SRSF6 overexpression. (B) Overlap of DEGs and RASGs following SRSF6 overexpression. (C and D) The top 10 representative (C) GO biological process terms and (D) KEGG pathways of RASGs. SRSF6, serine and arginine-rich splicing factor 6; AS, alternative splicing; DEG, differentially expressed gene; RASG, regulated AS gene; GO, gene ontology; KEGG, Kyoto Encyclopedia of Genes and Genomes; MXE, mutual exclusive exon skipping; 3pMXE, alternative last exon; 5pMXE, alternative first exon; A3SS, alternative 3' splice site; A5SS, alternative 5' splice site; cassetteExon, exon included; ES, exon skipping; IntronR, intron retention.

GEPIA2 (36), a web-based tool that compares gene expression between tumor and normal tissues from TCGA. SRSF6 expression in tumors had a higher median relative expression compared with that in normal tissues in 13 of the 16 cancer types (Fig. 1A). SRSF6 expression in tumors was significantly upregulated $(\mathrm{FDR}<0.05)$ in four cancer types and was significantly downregulated only in kidney chromophobe (KICH). Furthermore, GEPIA was used to comprehensively analyze the association of SRSF6 expression with survival rates in various types of cancer. As presented in Fig. 1B, the hazard ratios were significantly higher in $\mathrm{KICH}$ and liver hepatocellular carcinoma. These results suggested that SRSF6 is a marker for cancers and may have a role in tumorigenesis.

Overexpression of SRSF6 promotes HeLa cell apoptosis and inhibits cell growth in vitro. To investigate the function of SRSF6 in cancer, an SRSF6-overexpressing cell model was established by transfecting HeLa cells with an SRSF6-overexpression plasmid. It was verified that SRSF6 gene expression was significantly increased by RT-qPCR (Fig. 2A) and western blot analysis (Fig. 2B). To characterize the role of SRSF6 in regulating cell apoptosis and proliferation of HeLa cells, MTT assays and flow cytometric analyses were respectively performed. The results suggested that cell apoptosis was significantly increased in the SRSF6-OE group ( $\mathrm{P}<0.01$; Fig. $2 \mathrm{C}$ and $\mathrm{D})$; however, cell proliferation was significantly reduced in the SRSF6-OE group $(\mathrm{P}<0.01 ;$ Fig. $2 \mathrm{E})$. These results indicated that SRSF6 may be involved in regulating cell apoptosis and cell growth in HeLa cells.
SRSF6 overexpression induces transcriptional differences. To further investigate SRSF6-mediated transcriptional or post-transcriptional regulation, cDNA libraries with SRSF6-OE cells and control cells were constructed for RNA-seq on the Illumina HiSeq Xten platform. A total of two biological replicates were used and a total of 82.4-85.7 Million (M) 150-nucleotide paired-end raw reads per sample were obtained. After removing adaptors and low-quality reads, 75.2-82.1 M clean reads were aligned to the human GRCH38 genome using TopHat2, of which $87.15-91.42 \%$ were aligned and $93.19-96.53 \%$ were uniquely aligned (Table SII). The level of gene expression was calculated in the units of FPKM. A total of 27,378 expressed genes were assessed by RNA-seq. Effective overexpression of SRSF6 was further confirmed in parallel with the RNA-seq analysis (Fig. 3A).

The DEGs between the SRSF6-OE and control cells were determined using an absolute $\mathrm{FC} \geq 2$ and a $5 \%$ FDR as criteria with the edgeR package (42). A total of 422 upregulated and 515 downregulated DEGs were identified (Table SIII). The DEGs associated with SRSF6-OE are displayed in a volcano plot (Fig. 3B). The heatmap demonstrated distinctly different transcription profiles between the SRSF6-OE and control groups (Fig. 3C).

To further explore the potential biological roles of these DEGs, GO and KEGG enrichment analyses were performed. The top $10 \mathrm{GO}$ terms in the category biological process in SRSF6-OE cells, including up- or downregulated of genes, are presented in Fig. 3D (details in Table SIV). The upregulated genes in the SRSF6-OE cells were mainly enriched in proteolysis, blood coagulation, transport and negative 
A
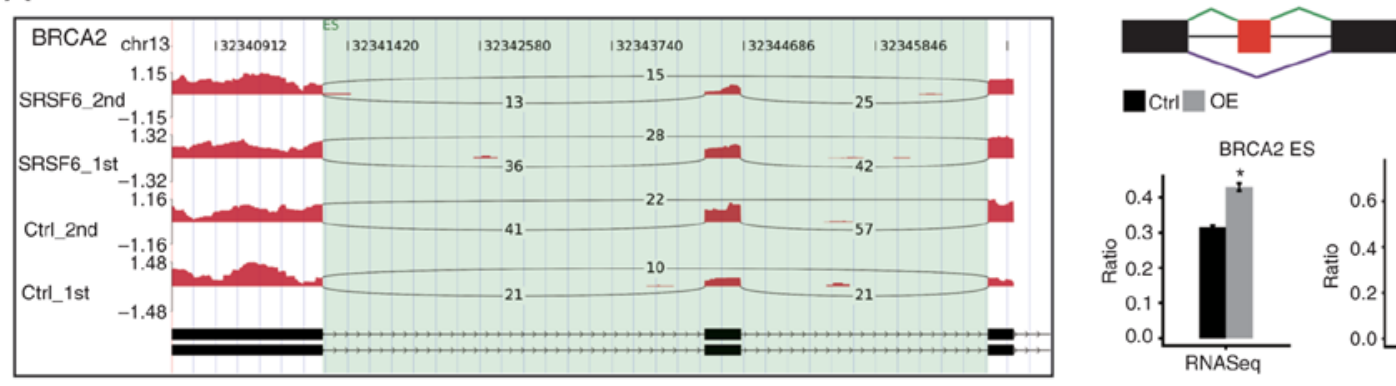

$\square \mathrm{Ctrl} \square \mathrm{OE}$

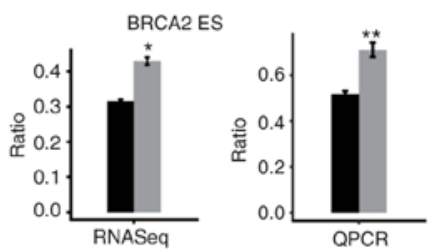

B
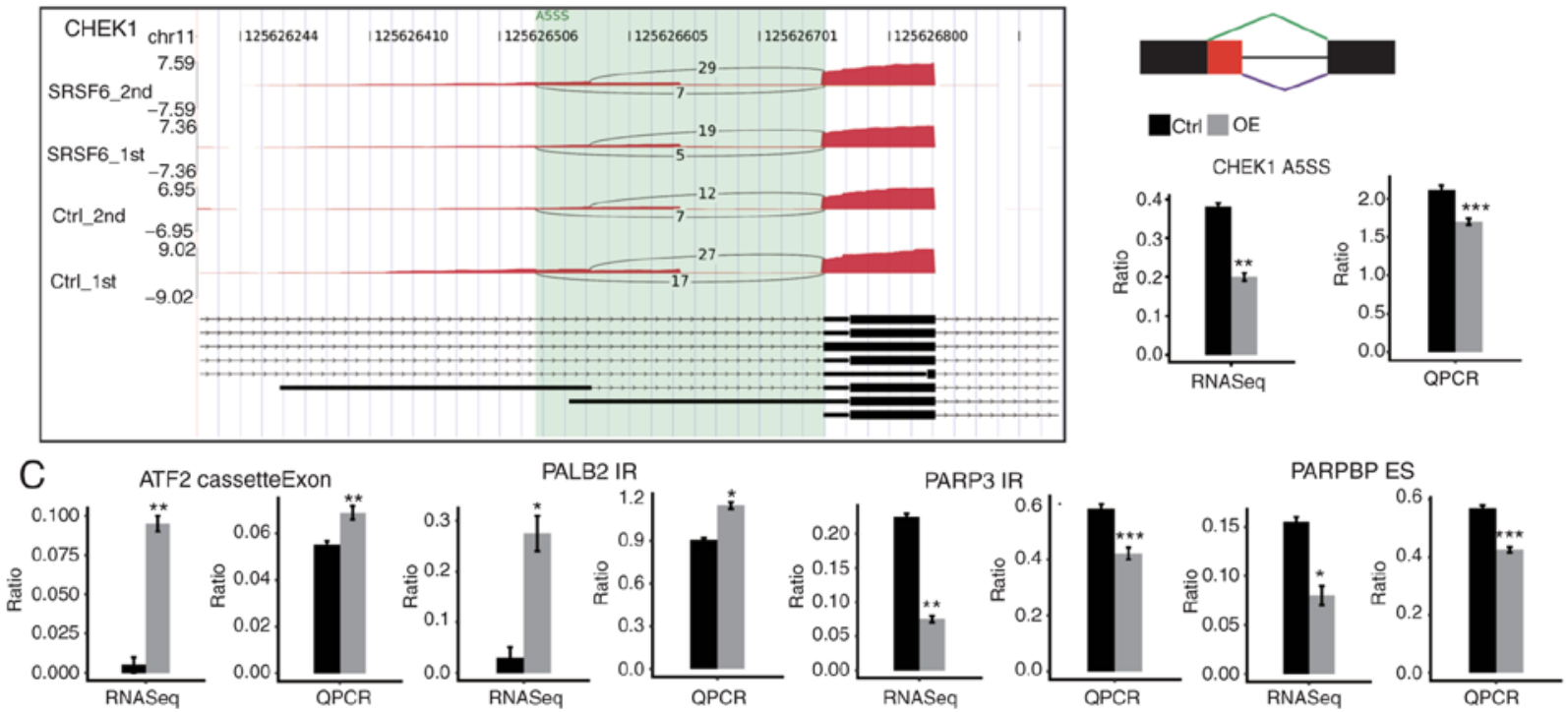

Figure 5. Validation of ASEs in cancer cells. (A and B) Genome visualization (left panel) indicates SRSF6-regulated ASEs in SRSF6-overexpression and control cells. (A) BRCA2 and (B) CHEK1. The number of junction reads was marked on the line representing splice junctions composing ASEs. The structures of the ASEs are depicted in the top-right panel. The altered ratio of ASEs according to RNA-seq and reverse transcription-qPCR was calculated and plotted (right panel, bottom). Ctrl_1st and Ctrl_2nd, SRSF6_1st and SRSF6_2nd are two biological replicates. (C) Validation results of the other four ASEs as in the right panels in A and B. Black bars are for the control group and grey bars for SRSF6 OE. ${ }^{*} \mathrm{P}<0.05,{ }^{* *} \mathrm{P}<0.01,{ }^{* * * *} \mathrm{P}<0.001$ vs. Ctrl. SRSF6, serine and arginine-rich splicing factor 6; OE, overexpression; Ctrl, control; chr, chromosome; qPCR, quantitative PCR; RNA-seq, RNA sequencing; CHEK1, checkpoint kinase 1; ASE, alternative splicing event; PARP3, poly(ADP-ribose) polymerase family member 3; PALB2, partner and localizer of BRCA2; CHEK1, checkpoint kinase 1; PARPBP, PARP1 binding protein; BRCA2, BRCA2 DNA repair associated; ATF2, activating transcription factor 2; ES, exon skipping; cassetteExon, exon included; IR, intron retention; A5SS, alternative 5' splice site.

regulation of transcription from RNA polymerase II promoter (Fig. 3D, upper panel). The downregulated genes were mostly associated with extracellular matrix organization, potassium ion transmembrane transport, axon guidance, extracellular matrix (ECM) disassembly, cell adhesion and platelet activation (Fig. 3D, lower panel). According to the KEGG analysis (details in Table SV), the pathways of the upregulated gene sets were mainly associated with the Wnt signaling pathway, circadian entrainment and the ECM-receptor interaction signaling pathway (Fig. 3E, upper panel). Downregulated gene sets were significantly enriched in ECM-receptor interaction, PI3K/Akt signaling pathway, protein digestion and absorption, and focal adhesion (Fig. 3E, lower panel). These results indicated that SRSF6 is important in regulating the cell cycle or ECM, which is directly associated with cellular quiescence, proliferation and cancer.

To confirm the important regulatory function of SRSF6 at the level of gene expression in the HeLa cell line, DEGs that were important in tumorigenesis were validated by RT-qPCR (Table SI). All of the seven validated DEGs after SRSF6 overexpression, including YTH N6-methyladenosine RNA binding protein 1, interferon induced protein with tetratricopeptide repeats 2 , RNA polymerase I subunit G, deleted in lymphocytic leukemia 2 like, protocadherin beta 14, RUN domain containing $3 \mathrm{~B}$ and keratin 13 , were confirmed (Fig. 3F).

SRSF6 significantly regulates AS of genes involved in DDR pathways. To investigate the regulatory role of SRSF6 in AS, the SRSF6-dependent ASEs were analyzed using the transcriptome sequencing data of HeLa cells. A total of $40.52-41.97 \%$ of uniquely mapped reads were spliced reads (Table SVI). Furthermore, 240,773 out of 367,321 annotated exons, as well as 158,256 annotated and 167,859 novel splice junctions were detected from the RNA-seq data. The ABLas software tool $(41,43)$ was then used to explore ASEs. A total of 19,404 known ASEs were detected, which were annotated in the reference genome and 61,995 novel ASEs were detected due to novel splice junctions (Table SVII).

By applying a stringent cut-off of $\mathrm{P} \leq 0.05$ and an AS ratio $\geq 0.2,661$ high-confidence RASEs were identified (Table SVIII). The RASEs included 76 CE, 92 ES, 109 A5SS, 91 A3SS, 13 cases of alternative last exon, 18 cases of 


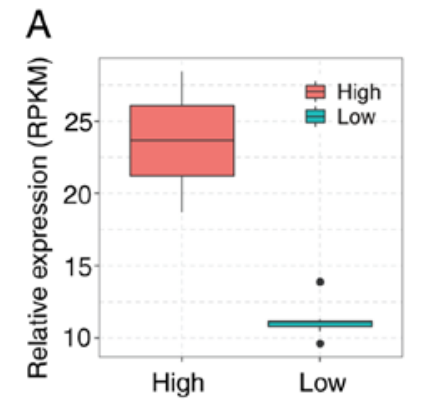

C

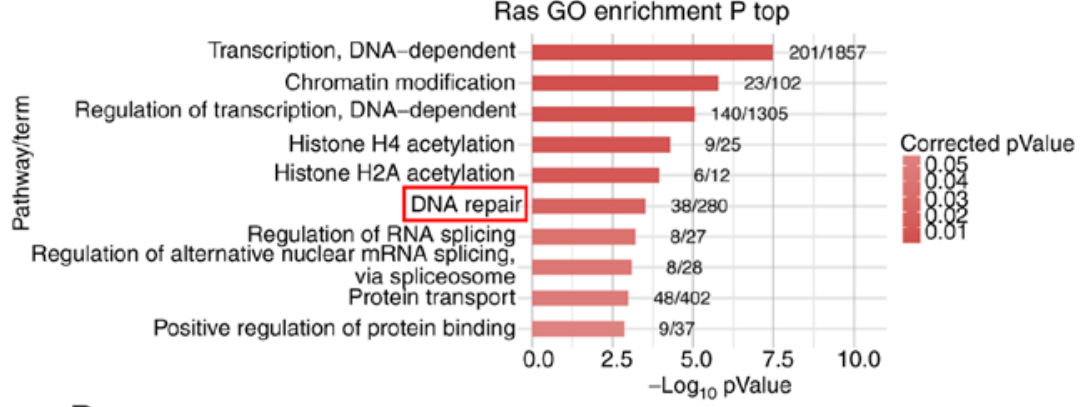

D

$\mathrm{B}$

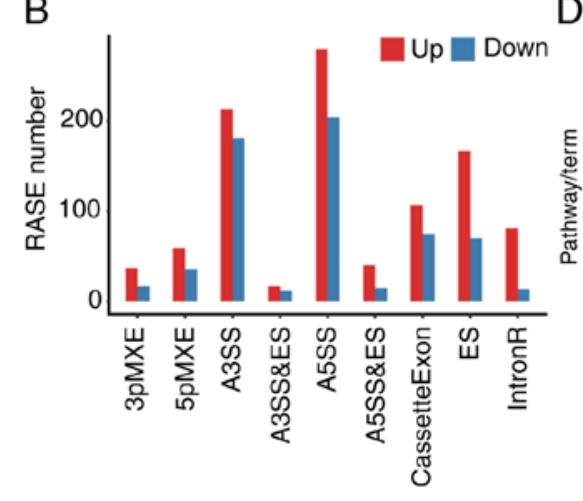

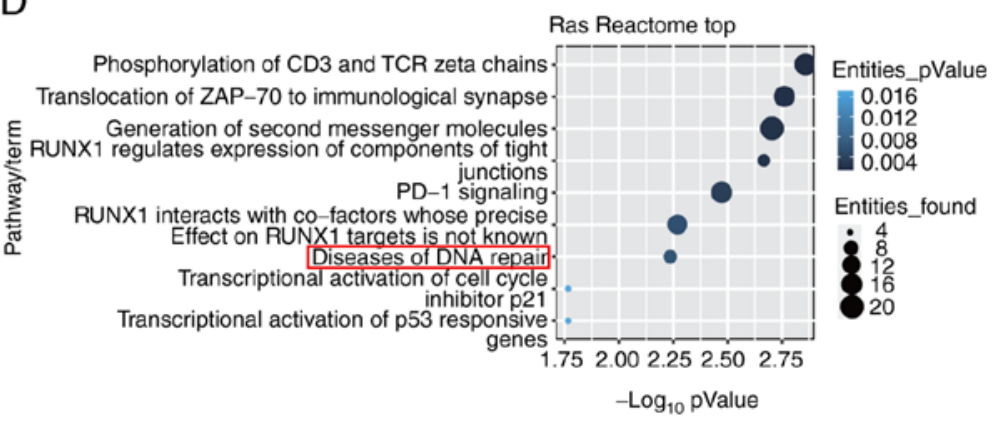

Figure 6. Analysis of potential SRSF6-regulated AS in cervical tumor samples. (A) A total of 16 tumor samples were divided into two groups based on the expression level of SRSF6. Box plots indicate the expression (RPKM) of SRSF6 in the two groups with high or low expression of SRSF6. The horizontal lines indicate the median, the boxes indicate the interquartile range and the vertical lines indicate the standard deviation. (B) Classification of regulated AS types in cervical tumors. (C) The top 10 representative GO biological process terms and (D) Reactome pathways in which RASGs were enriched following SRSF6 overexpression. SRSF6, serine and arginine-rich splicing factor 6; AS, alternative splicing; RASG, regulated AS gene; GO, gene ontology; RUNX1, RUNX family transcription factor 1; PD-1, programmed cell death 1; RPKM, reads per kilobase of transcript per million reads mapped; $3 \mathrm{pMXE}$, alternative last exon; 5pMXE, alternative first exon; A3SS, alternative 3' splice site; A5SS, alternative 5' splice site; cassetteExon, exon included; ES, exon skipping; IntronR, intron retention.

alternative first exon, 12 A3SS\&ES, 11 A5SS\&ES, 215 IR and 24 MXE (Fig. 4A). The data suggested that SRSF6 globally regulated ASEs in HeLa cells. Excluding the changes in ASEs attributed to transcriptional regulation, genes whose expression levels and AS were both regulated by SRSF6 were also examined and eight genes were shared between the DEGs and RASG: APC regulator of WNT signaling pathway 2, ATPase secretory pathway $\mathrm{Ca}^{2+}$ transporting 2, TBC/LysM-associated domain containing 2, HOXB cluster antisense RNA 4, RP11-18H7.1, AC005253.2, leucine rich repeat containing 24 and RP11-654A16.1 (Fig. 4B).

The AS genes that were identified by GO analysis were highly enriched in 'DNA repair', 'double-strand break repair via homologous recombination', 'DNA-dependent transcription', ' $\mathrm{G} 2 / \mathrm{M}$ transition of mitotic cell cycle' and 'response to DNA damage stimulus pathways' (GO biological process terms; Fig. 4C). The most enriched KEGG pathways included those involved in 'Selenocompound metabolism', 'Pyrimidine metabolism', 'Fanconi anemia pathway', 'Purine metabolism', 'Endometrial cancer', 'metabolism' and 'inflammatory related pathways' (Fig. 4D and Table SIX). Collectively, these results suggested that SRSF6 indeed regulated ASEs that were involved in pathways associated with the DDR, which is strongly associated with tumorigenesis (44).

Validation of SRSF6-regulated ASEs by RT-qPCR. According to the present results, SRSF6 regulated AS of genes primarily enriched in the DNA repair-related pathway. Therefore, nine genes involved in this pathway were selected and RT-qPCR assays were performed to validate the ASEs regulated by SRSF6. PCR primer pairs (Table SI) were designed to amplify the two different splicing isoforms (Model and AS) in these ASEs for nine genes. Among the nine events tested, the RT-qPCR results for six ASEs were consistent with the RNA-seq results, including two Intron Retention (IR), one A5SS, 2 ES and $1 \mathrm{CE}$ which were located on poly(ADP-ribose) polymerase family member (PARP) 3, BRCA2 DNA repair associated (BRCA2), partner and localizer of BRCA2, checkpoint kinase 1, PARP1 binding protein and activating transcription factor 2 (Fig. 5). The consistent validation results demonstrated the confidence in the SRSF6-regulated ASEs identified.

Analysis of the SRSF6-regulated AS network in cervical cancer samples. Since accumulating evidence has indicated a marked association between altered DDR and cancer progression and poor outcome $(44,45)$, it was next queried whether SRSF6-regulated ASEs identified in HeLa cells were also regulated by SRSF6 in clinical cancer samples. A total of 16 cervical cancer samples from TCGA were selected, 8 with high SRSF6 expression and 8 with low SRSF6 expression (Fig. 6A). A total of $170 \pm 99.4 \mathrm{M}$ clean reads per sample were downloaded from TCGA and 115.8 $\pm 71.4 \mathrm{M}$ reads per sample were uniquely aligned to the human genome, of which $8.72-15.08 \%$ belonged to junction reads (Table SX). By using the ABLas software tool, 34,465 known ASEs and 52,623 novel ASEs were detected (Table SXI). The same stringent cut-off ( $\mathrm{P} \leq 0.05$ 


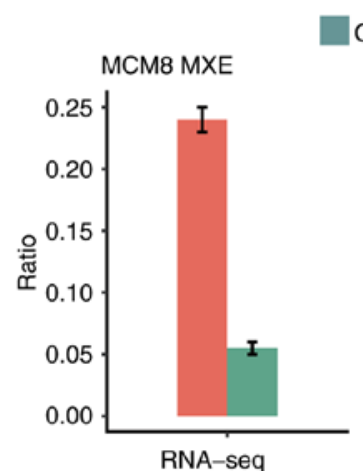

Con $\square \mathrm{OE}$
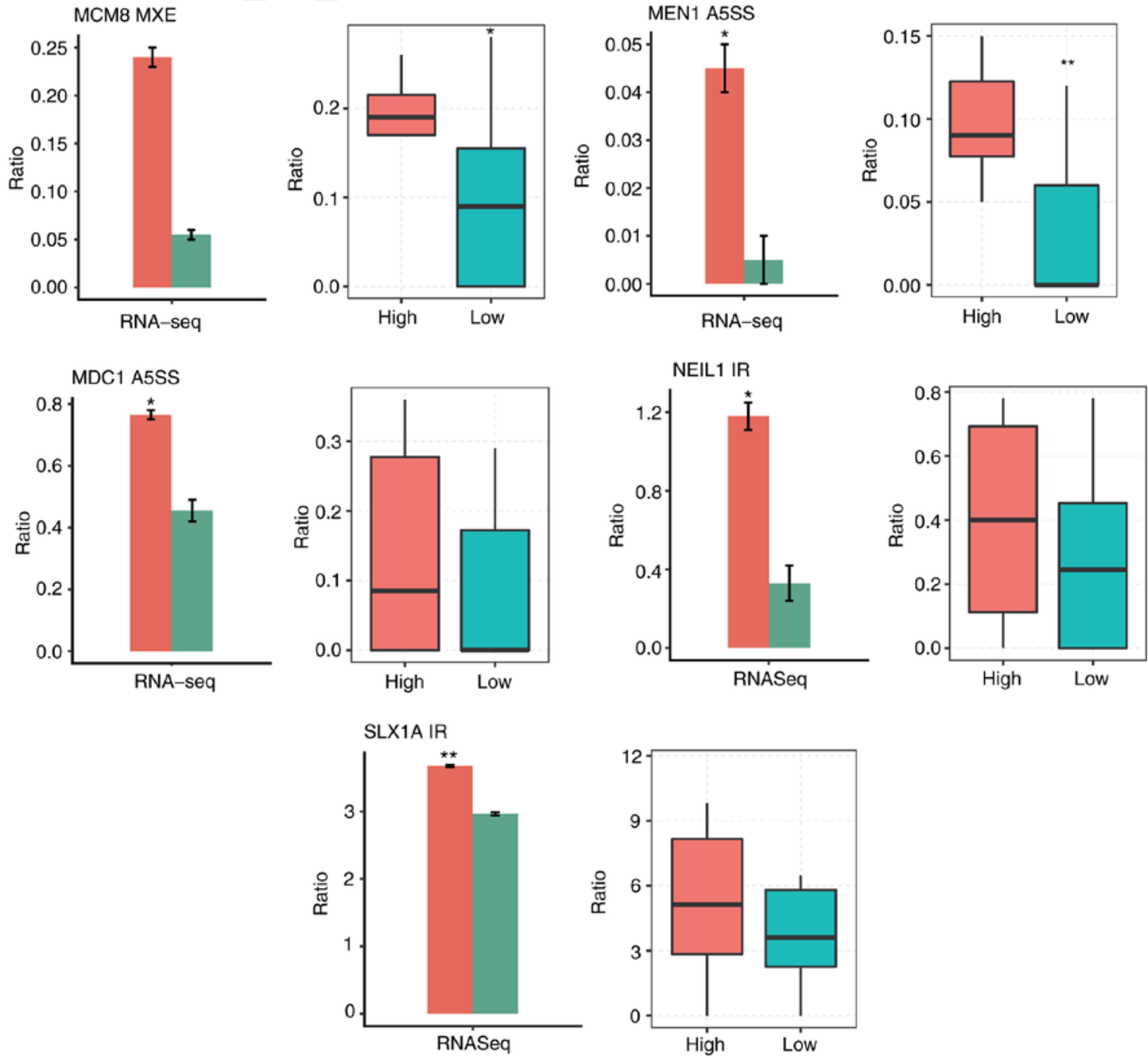

Figure 7. Comparison of regulated ASEs in cancer cells (left) and in tumor samples (right). Splicing ratio of ASEs involved in DNA damage response-associated terms, which were detected in cancer cell pathways, were compared. ${ }^{*} \mathrm{P}<0.05,{ }^{* *} \mathrm{P}<0.01$ vs. control. RNA-seq, RNA sequencing; Ctrl, control; OE, overexpression; ASE, alternative splicing event; MCM8, minichromosome maintenance 8 homologous recombination repair factor; MEN1, menin 1; MDC1, mediator of DNA damage checkpoint 1; NEIL1, nei-like DNA glycosylase 1; SLX1A, SLX1 homolog A, structure-specific endonuclease subunit; IR, intron retention; A5SS, alternative 5' splice site; MXE, mutual exclusive exon skipping.

and T-value $\geq 0$ ) was applied to identify RASEs with high confidence and a total of 2,225 SRSF6-regulated ASEs were obtained in clinical cancer samples (Table SXII), including 182 CE/237 ES, 484 A5SS, 394 A3SS and 647 IR (Fig. 6B). These results confirmed that SRSF6 is able to globally regulate the AS process in cervical cancer.

Further analysis of genes that had been alternatively spliced revealed that these genes were highly enriched in DNA-dependent regulation of transcription, chromatin modification, histone acetylation, DNA repair and RNA splicing pathways (GO biological process terms; Fig. 6C). Of note, biological pathways enriched in SRSF6-regulated AS in HeLa cells were similar to those in cervical cancer samples. These results highlighted again the robust function of splicing factor SRSF6 in regulating ASEs involved in different pathways of cancer, particularly the DNA repair pathway, which was the most representative and meaningful pathway. Reactome pathway analysis also revealed that SRSF6-regulated AS genes were enriched in pathways of 'Diseases of DNA repair' (Fig. 6D).
Furthermore, 9 significantly differential ASEs involved in the genes of DDR-associated terms detected in cancer cells were then compared with ASEs detected in 16 cervical cancer samples from TCGA. Of the 9 tested ASEs, five ASEs were consistent. The five validated splicing events were located in the following genes: Minichromosome maintenance 8 homologous recombination repair factor (MCM8), menin 1 (MEN1), mediator of DNA damage checkpoint 1 (MDC1), nei-like DNA glycosylase 1 (NEIL1) and SLX1 homolog A, structure-specific endonuclease subunit (SLX1A) (Fig. 7). To summarize these results, SRSF6 appears to have a significant role in cancer progression by regulating AS of important cancer-associated genes and pathways, particularly DDR pathways.

\section{Discussion}

In the present study, the role of SRSF6 in HeLa cells was investigated and it was explored how SRSF6 regulates AS. First, using GEPIA, it was revealed that 13 out of 16 cancer types from 
TCGA exhibited upregulation of SRSF6, suggesting that SRSF6 may have an important role in tumor development. Based on this premise, the HeLa cell line was used as a model to analyze the consequences of overexpression of SRSF6. Overexpression of SRSF6 promoted cell apoptosis and inhibited cell proliferation. At the cellular level, it was observed that overexpression of SRSF6 had a broad effect on gene expression and functional clusters of DEGs highly enriched in cancer-associated terms were obtained. Furthermore, it was observed that overexpression of SRSF6 significantly regulated AS of genes involved in the DDR pathway. These results revealed a role for SRSF6 in transcriptional and post-transcriptional regulation during cancer progression. The data of clinical samples were then analyzed and 16 cervical cancer samples from TCGA were selected, including 8 with high SRSF6 expression and 8 displaying low SRSF6 expression, to further study the potential impact of SRSF6 on AS regulation of the cancer transcriptome. Significant differential ASEs in these two groups were involved in different pathways in cervical carcinoma, with the DNA repair pathway being the most representative.

Previous studies reported that SRSF6 was commonly upregulated in lung (28), colon (28), skin (29) and colorectal (30) cancers. SRSF6 overexpression synergizes with MYC and its upregulation promotes the transformation of lung epithelial cells and may trigger abnormal proliferation (28). In the present study, GEPIA was used to analyze data from TCGA and the results suggested that 13 of 16 cancer types from TCGA exhibited higher levels of SRSF6 in most types of tumor compared with matched normal tissues, while 3 of 16 cancer types (KICH-kidney chromophobe, KIRC-kidney renal clear-cell carcinoma and THCA-thyroid carcinoma) had lower expression levels of SRSF6. Of note, in an in vitro experiment of the present study, overexpression of SRSF6 in HeLa cells was indicated to promote cell apoptosis and inhibit cell proliferation, which contradicts the results in colorectal and lung cancer. Although oncogenes are more likely to be overexpressed and tumor suppressor genes are more frequently disrupted, certain genes have oncogenic and tumor-suppressor functions in different tumor types or even within the natural evolution of the disease in a single tumor (46). These conflicting roles are a result of the complexity of biological pathways, the heterogeneity of cancer cells and the higher network degrees of the gene $(46,47)$. In the present study, it was speculated that SRSF6 may act as a tumor suppressor in certain cancer types, including $\mathrm{KICH}$, cervical squamous-cell carcinoma and endocervical adenocarcinoma.

DDR is an essential function in the maintenance of genome stability (48). When normal repair procedures fail, irreversible DNA damage may occur and uncontrolled cell division may lead to the formation of tumors or cancers $(49,50)$. Recent research on the genomic landscape of prostate cancer has revealed that a significant number of cases harbor DDR genetic aberrations, with BRCA2 as the most common altered gene $(6-8,51)$. Molecular analysis of 333 primary prostate tumors identified deleterious aberrations in DDR genes, including BRCA2, BRCA1, cyclin-dependent kinase 12, ATM serine/threonine kinase, FA complementation group D2 and RAD51 paralog C, in 19\% of cases (62/333) (7). In this study, an in vitro model of SRSF6 overexpression was constructed and RNA-seq analysis was performed, including DEG and AS analysis. A certain variation among biological replicates of SRSF6-OE or Ctrl samples was observed. This was most likely due to a lack of sufficient repetition. Biological replicates are absolutely essential for differential expression analysis. However, in general, biological replicates tend to have more variability than technical replicates and it is particularly difficult to determine for cell lines. More biological repetitions provide a better estimate of biological variation and a more accurate estimate of average expression levels (52). A total of 18 significant ASEs involved in DNA repair were filtered out. Next, RNA-seq data in cancer cells were compared with RNA-seq data in clinical samples and five DDR-associated genes with a consistent response were identified: MCM8, MEN1, MDC1, NEIL1 and SLX1. These five genes are generally linked to tumorigenesis and progression, including cell proliferation, apoptosis and genome stability (53-58). Their downstream targets are regulated by SRSF6 and may influence cancer progression in cells or in clinical samples.

Dysregulation of the cell cycle machinery causes dysregulation of cell division, inducing cancer development. To drive the cell cycle properly, expression levels of cell cycle regulators are tightly regulated throughout the cell cycle. MCM8 and MCM9 are paralogues of the MCM2-7 eukaryotic DNA replication helicase complex proteins. It is increasingly recognized that MCM8 and MCM9 are involved in HR repair as a heterohexameric MCM8-9 complex (59-61). Mutations of numerous helicases are directly implicated in genetic diseases, including cancer and rapid aging. MCM8/9 were recently added to the catalog of helicases and mutations in MCM8/9, which correlate principally with primary ovarian failure/insufficiency and infertility, indicating a meiotic defect (56). MEN1 encodes menin, a tumor suppressor associated with a syndrome known as multiple endocrine neoplasia type I (57). Menin is a scaffold protein that functions in histone modification and epigenetic gene regulation. Menin specifically interacts with FANCD2, a protein encoded by a gene involved in DNA repair that has a critical role in repair of DNA damage (62). Interaction with NF-кB proteins and modulation of NF- $\mathrm{KB}$ transactivation contribute to the function of Menin as a tumor suppressor (63). However, further investigations are required to confirm and validate the association between SRSF6 and DDR, e.g. whether SRSF6-OE is able to induce DNA damage by using the comet assay.

In conclusion, the present study highlighted the functional importance of SRSF6 in mediating cancer progression by regulating AS. It was demonstrated that both in cancer cells and clinical tumor samples, SRSF6 regulates AS in genes enriched in DDR-associated functions and pathways. To elucidate the contribution of SRSF6 to cervical cancer, a series of analyses were performed and six novel genes that may be useful for further cancer research were isolated.

\section{Acknowledgements}

The authors thank International Science Editing (http://www. internationalscienceediting.com) for editing this manuscript.

\section{Funding}

The present study was supported by grants from the Preferential Projects supported by the Science and Technology Department of Jilin province (grant no. 20191102012YY). 


\section{Availability of data and materials}

The datasets used and/or analyzed during the current study are available under the gene expression omnibus series accession no. GSE138636.

\section{Authors' contributions}

$\mathrm{XY}$ and BS initiated the study, conceived the experiments and managed the project. XY, MW and BS wrote the manuscript. HJ, SF, PZ, WT, MW and CC performed the experiments and analyzed the data. All authors read and approved the final version of the manuscript.

\section{Ethics approval and consent to participate}

Not applicable.

\section{Patient consent for publication}

Not applicable.

\section{Competing interests}

The authors declare that they have no competing interests.

\section{References}

1. Bray F, Ferlay J, Soerjomataram I, Siegel RL, Torre LA and Jemal A: Global cancer statistics 2018: GLOBOCAN estimates of incidence and mortality worldwide for 36 cancers in 185 countries. CA Cancer J Clin 68: 394-424, 2018.

2. DePinho RA: The age of cancer. Nature 408: 248-254, 2000.

3. Preston DL, Mattsson A, Holmberg E, Shore R, Hildreth NG and Boice JD Jr: Radiation effects on breast cancer risk: A pooled analysis of eight cohorts. Radiat Res 158: 220-235, 2002.

4. Barbieri CE, Baca SC, Lawrence MS, Demichelis JF, Blattner M, Theurillat JP, White TA, Stojanov P, Van Allen E, Stransky N, et al: Exome sequencing identifies recurrent SPOP, FOXA1 and MED12 mutations in prostate cancer. Nat Genet 44: 685-689, 2012.

5. Kaczkowski B, Tanaka Y, Kawaji H, Sandelin A, Andersson R, Itoh M, Lassmann T, Hayashizaki Y, Carninci P and Forrest AR; FANTOM5 Consortium: Transcriptome analysis of recurrently deregulated genes across multiple cancers identifies new pan-cancer biomarkers. Cancer Res 76: 216-226, 2016.

6. Robinson D, Van Allen EM, Wu YM, Schultz N, Lonigro RJ, Mosquera JM, Montgomery B, Taplin ME, Pritchard CC, Attard G, et al: Integrative clinical genomics of advanced prostate cancer. Cell 161: 1215-1228, 2015.

7. Cancer Genome Atlas Research Network: The molecular taxonomy of primary prostate cancer. Cell 163: 1011-1025, 2015.

8. Grasso CS, Wu YM, Robinson DR, Cao X, Dhanasekaran SM, Khan AP, Quist MJ, Jing X, Lonigro RJ, Brenner JC, et al: The mutational landscape of lethal castration-resistant prostate cancer. Nature 487: 239-243, 2012

9. Hanahan D and Weinberg RA: Hallmarks of cancer: The next generation. Cell 144: 646-674, 2011.

10. Lord CJ and Ashworth A: The DNA damage response and cancer therapy. Nature 481: 287-294, 2012.

11. Shkreta L and Chabot B: The RNA splicing response to DNA damage. Biomolecules 5: 2935-2977, 2015.

12. Mata J, Marguerat S and Bähler J: Post-transcriptional control of gene expression: A genome-wide perspective. Trends Biochem Sci 30: 506-514, 2005

13. Venables JP: Aberrant and alternative splicing in cancer. Cancer Res 64: 7647-7654, 2004.

14. Wang ET, Sandberg R, Luo S, Khrebtukova I, Zhang L, Mayr C, Kingsmore SF, Schroth GP and Burge CB: Alternative isoform regulation in human tissue transcriptomes. Nature 456: 470-476, 2008.
15. David CJ and Manley JL: Alternative pre-mRNA splicing regulation in cancer: Pathways and programs unhinged. Genes Dev 24: 2343-2364, 2010.

16. Srebrow A and Kornblihtt AR: The connection between splicing and cancer. J Cell Sci 119: 2635-2641, 2006.

17. Ward AJ and Cooper TA: The pathobiology of splicing. J Pathol 220: 152-163, 2010.

18. Skotheim RI and Nees M: Alternative splicing in cancer: Noise, functional, or systematic? Int J Biochem Cell Biol 39: 1432-1449, 2007.

19. Howe D and Lynas C: The cyclin D1 alternative transcripts [a] and [b] are expressed in normal and malignant lymphocytes and their relative levels are influenced by the polymorphism at codon 241. Haematologica 86: 563-569, 2001.

20. Knudsen KE, Diehl JA, Haiman CA and Knudsen ES: Cyclin D1: Polymorphism, aberrant splicing and cancer risk. Oncogene 25: 1620-1628, 2006.

21. Comstock CE, Augello MA, Benito RP, Karch J, Tran TH, Utama FE, Tindall EA, Wang Y, Burd CJ, Groh EM, et al: Cyclin D1 splice variants: Polymorphism, risk, and isoform-specific regulation in prostate cancer. Clin Cancer Res 15: 5338-5349, 2009.

22. Wu JY and Maniatis T: Specific interactions between proteins implicated in splice site selection and regulated alternative splicing. Cell 75: 1061-1070, 1993.

23. Kohtz JD, Jamison SF, Will CL, Zuo P, Lührmann R, Garcia-Blanco MA and Manley JL: Protein-protein interactions and 5'-splice-site recognition in mammalian mRNA precursors Nature 368: 119-124, 1994.

24. Long JC and Caceres JF: The SR protein family of splicing factors: Master regulators of gene expression. Biochem J 417: $15-27,2009$.

25. Liu J, Huang B, Xiao Y, Xiong HM, Li J, Feng DQ, Chen XM, Zhang HB and Wang XZ: Aberrant expression of splicing factors in newly diagnosed acute myeloid leukemia. Onkologie 35: 335-340, 2012 .

26. Zou L, Zhang H, Du C, Liu X, Zhu S, Zhang W, Li Z, Gao C, Zhao X, Mei M, et al: Correlation of SRSF1 and PRMT1 expression with clinical status of pediatric acute lymphoblastic leukemia. J Hematol Oncol 5: 42, 2012.

27. Anczuków O, Akerman M, Cléry A, Wu J, Shen C, Shirole NH, Raimer A, Sun S, Jensen MA, Hua Y, et al: SRSF1-Regulated alternative splicing in breast cancer. Mol Cell 60: 105-117, 2015.

28. Cohen-Eliav M, Golan-Gerstl R, Siegfried Z, Andersen CL, Thorsen K, Ørntoft TF, Mu D and Karni R: The splicing factor SRSF6 is amplified and is an oncoprotein inlung and colon cancers. J Pathol 229: 630-639, 2013.

29. Jensen MA, Wilkinson JE and Krainer AR: Splicing factor SRSF6 promotes hyperplasia of sensitized skin. Nat Struct Mol Biol 21: 189-197, 2014

30. Wan L, Yu W, Shen E, Sun W, Liu Y, Kong J, Wu Y, Han F, Zhang L, Yu T, et al: SRSF6-regulated alternative splicing that promotes tumour progression offers a therapy target for colorectal cancer. Gut 68: 118-129, 2019.

31. Karni R, de Stanchina E, Lowe SW, Sinha R, Mu D and Krainer AR: The gene encoding the splicing factor SF2/ASF is a proto-oncogene. Nat Struct Mol Biol 14: 185-193, 2007.

32. Anczukow O, Rosenberg AZ, Akerman M, Das S, Zhan L, Karni R, Muthuswamy SK and Krainer AR: The splicing factor SRSF1 regulates apoptosis and proliferation to promote mammary epithelial cell transformation. Nat Struct Mol Biol 19: 220-228, 2012.

33. Jia R, Li C, McCoy JP, Deng CX and Zheng ZM: SRp20 is a proto-oncogene critical for cell proliferation and tumor induction and maintenance. Int J Biol Sci 6: 806-826, 2010.

34. Fernando C, Audibert A, Simon F, Tazi J and Juge F: A role for the serine/arginine-rich (SR) protein B52/SRSF6 in cell growth and myc expression in drosophila. Genetics 199: 1201-1513, 2015.

35. Kong J, Sun W, Li C, Wan L, Wang S, Wu Y, Xu E, Zhang H and La M: Long non-coding RNA LINC01133 inhibits epithelial-mesenchymal transition and metastasis in colorectal cancer by interacting with SRSF6. Cancer Lett 380: 476-484, 2016.

36. Tang Z, Li C, Kang B, Gao G, Li C and Zhang Z: GEPIA: A web server for cancer and normal gene expression profiling and interactive analyses. Nucleic Acids Res 45: W98-W102, 2017.

37. Tu Y, Wu X, Yu F, Dang J, Wei Y, Yu H, Liao W, Zhang Y and Wang J: Tristetraprolin-RNA interaction map reveals a novel TTP-RelB regulatory network for innate immunity gene expression. Mol Immunol 121: 59-71, 2020.

38. Livak KJ and Schmittgen TD: Analysis of relative gene expression data using real-time quantitative PCR and the 2(-Delta Delta C(T)) method. Methods 25: 402-408, 2001. 
39. Kim D, Pertea G, Trapnell C, Pimentel H, Kelley R and Salzberg SL: TopHat2: Accurate alignment of transcriptomes in the presence of insertions, deletions and gene fusions. Genome Biol 14: R36, 2013.

40. Robinson MD, McCarthy DJ and Smyth GK: EdgeR: A bioconductor package for differential expression analysis of digital gene expression data. Bioinformatics 26: 139-140, 2010.

41. Xia H, Chen D, Wu Q, Wu G, Zhou Y, Zhang Y and Zhang L: CELF1 preferentially binds to exon-intron boundary and regulates alternative splicing in heLa cells. Biochim Biophys Acta Gene Regul Mech 1860: 911-921, 2017.

42. Xie C, Mao X, Huang J, Ding Y, Wu J, Dong S, Kong L, Gao G, Li CY and Wei L: KOBAS 2.0: A web server for annotation and identification of enriched pathways and diseases. Nucleic Acids Res 39: W316-W322, 2011.

43. Jin L, Li G, Yu D, Huang W, Cheng C, Liao S, Wu Q and Zhang Y: Transcriptome analysis reveals the complexity of alternative splicing regulation in the fungus verticillium dahliae. BMC Genomics 18: 130, 2017.

44. Nombela P, Lozano R, Aytes A, Mateo J, Olmos D and Castro E: BRCA2 and other DDR genes in prostate cancer. Cancers (Basel) 12: 352, 2019.

45. Schiewer MJ and Knudsen KE: DNA damage response in prostate cancer. Cold Spring Harb Perspect Med 2: a030486, 2019.

46. Shen L, Shi Q and Wang W: Double agents: Genes with both oncogenic and tumor-suppressor functions. Oncogenesis 13: 25 , 2018.

47. Barros-Filho MC, Guisier F, Rock LD, Becker-Santos DD, Sage AP, Marshall EA and Lam WL: Tumour suppressor genes with oncogenic roles in lung cancer. IntechOpen, 2019. https://www. intechopen.com/books/genes-and-cancer/tumour-suppressor-geneswith-oncogenic-roles-in-lung-cancer. Accessed April 16, 2019.

48. Yoshida K and Miki Y: Role of BRCA1 and BRCA2 as regulators of DNA repair, transcription, and cell cycle in response to DNA damage. Cancer Sci 95: 866-871, 2004

49. Helleday T, Petermann E, Lundin C, Hodgson B and Sharma RA DNA repair pathways as targets for cancer therapy. Nat Rev Cancer 8: 193-204, 2008.

50. Jeggo PA, Pearl LH and Carr AM: DNA repair, genome stability and cancer: A historical perspective. Nat Rev Cancer 16: 35 , 2016.

51. Kumar A, Coleman I, Morrissey C, Zhang X, True LD, Gulati R, Etzioni R, Bolouri H, Montgomery B, White T, et al: Substantial interindividual and limited intraindividual genomic diversity among tumors from men with metastatic prostate cancer. Nat Med 22: 369-378, 2016.

52. Klaus B: Statistical relevance-relevant statistics, part I. EMBO J 34: 2727-2730, 2015
53. Ji X, Li J, Zhu L, Cai J, Zhang J, Qu Y, Zhang H, Liu B, Zhao R and Zhu Z: CHD1L promotes tumor progression and predicts survival in colorectal carcinoma. J Surg Res 185: 84-91, 2013.

54. Nihira NT and Yoshida K: Engagement of DYRK2 in proper control for cell division. Cell Cycle 14: 802-807, 2015

55. Sailo BL, Banik K, Girisa S, Bordoloi D, Fan L, Halim CE, Wang H, Kumar AP, Zheng D, Mao X, et al: FBXW7 in cancer: What has been unraveled thus far? Cancers (Basel) 19: 246, 2019.

56. Griffin WC and Trakselis MA: The MCM8/9 complex: A recent recruit to the roster of helicases involved in genome maintenance. DNA Repair (Amst) 76: 1-10, 2019.

57. Kamilaris CD and Stratakis CA: Multiple endocrine neoplasia type 1 (MEN1): An update and the significance of early genetic and clinical diagnosis. Front Endocrinol (Lausanne) 10: 339, 2019.

58. Peng J, Tang L, Cai M, Chen H, Wong J and Zhang P: RECQL5 plays an essential role in maintaining genome stability and viability of triple-negative breast cancer cells. Cancer Med 8: 4743-4752, 2019

59. Lutzmann M, Grey C, Traver S, Ganier O, Maya-Mendoza A, Ranisavljevic N, Bernex F, Nishiyama A, Montel N, Gavois E, et al: MCM8- and MCM9-deficient mice reveal gametogenesis defects and genome instability due to impaired homologous recombination. Mol Cell 47: 523-534, 2012.

60. Nishimura K, Ishiai M, Horikawa K, Fukagawa T, Takata M, Takisawa $\mathrm{H}$ and Kanemaki MT: Mcm8 and Mcm9 form a complex that functions in homologous recombination repair induced by DNA interstrand crosslinks. Mol Cell 47: 511-522, 2012.

61. Park J, Long DT, Lee KY, Abbas T, Shibata E, Negishi M, Luo Y, Schimenti JC, Gambus A, Walter JC and Dutta A: The MCM8-MCM9 complex promotes RAD51 recruitment at DNA damage sites to facilitate homologous recombination. Mol Cell Biol 33: 1632-1644, 2013.

62. Jin S, Mao H, Schnepp RW, Sykes SM, Silva AC, D'Andrea AD and Hua X: Menin associates with FANCD2, a protein involved in repair of DNA damage. Cancer Res 63: 4204-4210, 2003.

63. Heppner C, Bilimoria KY, Agarwal SK, Kester M, Whitty LJ, Guru SC, Chandrasekharappa SC, Collins FS, Spiegel AM, Marx SJ and Burns AL: The tumor suppressor protein menin interacts with NF-kappaB proteins and inhibits NF-kappaB-mediated transactivation. Oncogene 20: 4917-4925, 2001.

This work is licensed under a Creative Commons Attribution-NonCommercial-NoDerivatives 4.0 International (CC BY-NC-ND 4.0) License. 